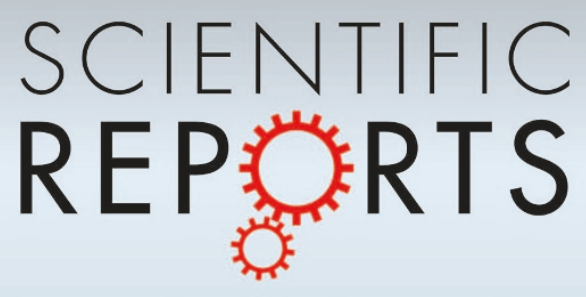

OPEN

SUBJECT AREAS:

NEUROINFORMATICS

INFORMATION TECHNOLOGY

Received

28 November 2013

Accepted

9 April 2014

Published

15 May 2014

Correspondence and requests for materials should be addressed to H.G. (huigong@mail. hust.edu.cn) or S.Q.Z.

(sqzeng@mail.hust. edu.cn)

* These authors contributed equally to this work.

\title{
Digital reconstruction of the cell body in dense neural circuits using a spherical-coordinated variational model
}

\author{
Tingwei Quan ${ }^{1,2,3 *}$, Jing $\mathrm{Li}^{1,2 *}$, Hang Zhou ${ }^{1,2}$, Shiwei $\mathrm{Li}^{1,2}$, Ting Zheng ${ }^{1,2}$, Zhongqing Yang ${ }^{1,2}$, \\ Qingming Luo ${ }^{1,2}$, Hui Gong ${ }^{1,2}$ \& Shaoqun Zeng ${ }^{1,2}$
}

\begin{abstract}
'Britton Chance Center for Biomedical Photonics, Huazhong University of Science and Technology-Wuhan National Laboratory for Optoelectronics, Wuhan 430074, China, ${ }^{2}$ MoE Key Laboratory for Biomedical Photonics, Department of Biomedical Engineering, Huazhong University of Science and Technology, Wuhan 430074, China, ${ }^{3}$ School of Mathematics and Statistics, Hubei University of Education, Wuhan 430205, China.
\end{abstract}

Mapping the neuronal circuits is essential to understand brain function. Recent technological advancements have made it possible to acquire the brain atlas at single cell resolution. Digital reconstruction of the neural circuits down to this level across the whole brain would significantly facilitate brain studies. However, automatic reconstruction of the dense neural connections from microscopic image still remains a challenge. Here we developed a spherical-coordinate based variational model to reconstruct the shape of the cell body i.e. soma, as one of the procedures for this purpose. When intuitively processing the volumetric images in the spherical coordinate system, the reconstruction of somas with variational model is no longer sensitive to the interference of the complicated neuronal morphology, and could automatically and robustly achieve accurate soma shape regardless of the dense spatial distribution, and diversity in cell size, and morphology. We believe this method would speed drawing the neural circuits and boost brain studies.

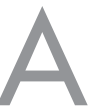

ccurately drawing and quantitatively describing the map of neuronal circuits play an important role in understanding how brain works ${ }^{1-3}$. Digital reconstruction of neuronal morphology from image stacks is essential and indispensable for drawing the neuronal circuits ${ }^{4-6}$. Basically the connectivity between neurons can be inferred by digitizing the morphology of somas and dendrites, and then detecting the structural connections $^{7-11}$. Recent progresses in molecular fluorescence labeling ${ }^{12}$ and Golgi staining technique ${ }^{13}$ and highthroughput imaging techniques ${ }^{14-22}$ allow imaging a mouse brain at micron or even submicron resolution, which in principle provide huge image datasets with adequate details to reveal the architecture ranging from one single neuron to a comprehensive brain-wide neural circuits. However, digitizing the morphology of neurons from these huge volume digital datasets is extreme difficult and appears as a bottle neck at neuronal level connectivity studies. One of the major obstacles is that in high resolution images, neuron and its fibers (called process) are too dense to perform an automatic segmentation for digital reconstruction. Shape reconstruction of somas, as an important part of the digital reconstruction, still remains a challenge. And this mainly results from the complexity of neuronal images, namely the huge variation in soma distribution density, soma size as well as the morphology of dendrites, all of which make it very difficult to describe the neurons using a single pattern.

There are many methods developed to segment and reconstruct the shape of cells effectively from the microscopic images, such as watershed based methods ${ }^{23-29}$, active contour based methods ${ }^{30-34}$, gradient flow tracking algorithm ${ }^{35}$, concavity detection methods $\mathrm{s}^{36,37}$, voronoi diagrams methods ${ }^{38,39}$, and the graph methods ${ }^{40-42}$, all of which are able to accurately segment and reconstruct the shape of cells, each for specific purpose. However, none of these methods involve the reconstruction of soma's shape under the severe influence of dense and thick neuronal trucks. For this application, rayburst sampling method ${ }^{43-46}$ has been proposed as a good tool for shape reconstruction of single neuron, and it works well in the segmentation of somas, dendrites, and even spines. However, they are not applicable to reconstruct the shape of clustered somas. The major obstacle here is that rays cast from the center point of a soma fail to end at the right soma boundary, instead they tend to wrongly end at the contacted adjacent soma boundary as designed to be stopped by the intensity threshold.

In contrast to rayburst sampling method ${ }^{43}$, active contour model ${ }^{47,48}$ might avoid this problem. By minimizing the designed variational model, active contour model can make the initial boundary elements move to the boundary where the biggest differences of signal intensity appear. Also, it is required that the boundary formed 


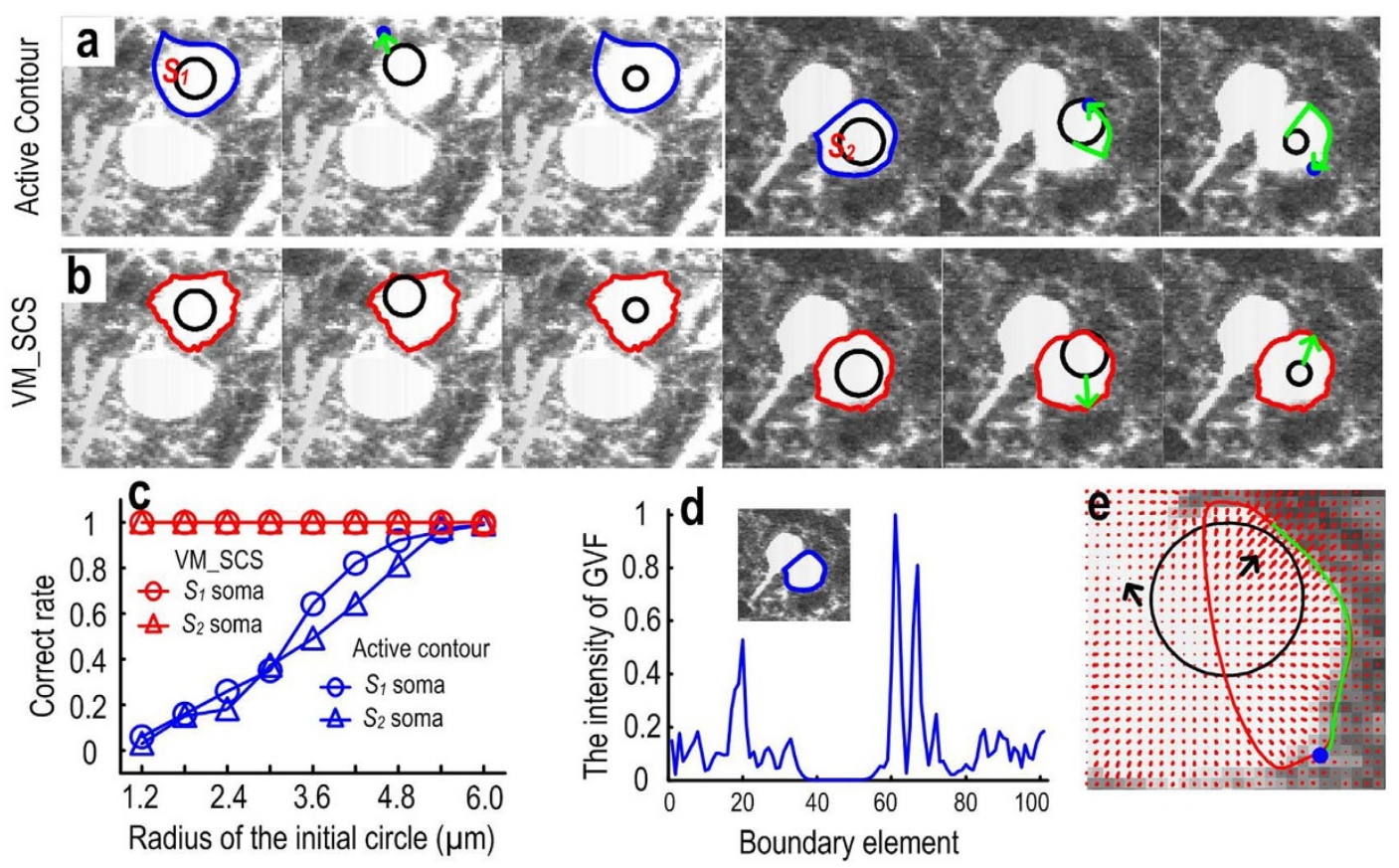

Figure 1 Comparisons of the robustness of VM_SCS and active contour model to the initial boundary. (a) Using active contour model to reconstruct the shape of the separately and closely positioned somas, the reconstructions (blue curves or blue dots) were largely determined by the initial boundaries (black circles). (b) With the same data and initial boundaries (black circles) in (a), almost identical curves (read curves) can be reconstructed using VM_SCS. The green curved (a) or the straight arrow (b) denoted as the trajectory that a boundary element moved. (c) The rate of accurate reconstructions of VM_SCS and active contour model were compared when the initial boundaries were set to the circles with the random centers and radius ranging from $1.2 \mu \mathrm{m}$ to $6 \mu \mathrm{m}$. (d) The intensity of gradient vector flow as a function of boundary elements that formed the boundary. The boundary was labeled by the blue curve in the embedded image. (e) Low isotropy of gradient vector flow makes the initial boundary (black circle) move to the red curve, the green curve and finally the blue dot.

by the moving elements is smooth, preventing some elements from arriving on the boundary of other somas. However, active contour model still experiences difficulties in processing some complex images. The reason, in most cases, is that the low isotropy of gradient vector flow (GVF) in the images causes all boundary elements moving towards a point. GVF here is regarded as the smoothing gradient of the boundary map pointing towards the boundary. Restricting moving directions of boundary elements may eliminate the effect of low isotropy of GVF. Inspired by this idea, here we have developed a special variational model, termed as VM_SCS (variation model based on sphere coordinate system), to reconstruct neuronal soma shape free from the influence of thick trucks in dense neural circuits. In this method, we convert the volumetric image data from the original Cartesian coordinate system to the sphere coordinate system. With the advantage of the sphere coordinate system, for each boundary element, its moving direction can be fixed by a preset polar and azimuth angle. This strategy decreases the dependence of our model on the initial boundary conditions, and thus provides a high robustness to the setting of the initial boundary. In addition, our model possesses the advantages of active contour model ${ }^{47,48}$. So our model can also accurately reconstruct the shapes of the clustered neuronal somas, and eliminate the interference of the thick dendritic trucks on soma shape reconstruction. With VM_SCS, the shape of neuronal somas with different size is also effectively reconstructed.

\section{Results}

The robustness of VM_SCS to the initial boundary. As active contour method ${ }^{48}$ is often frustrated by the initial boundary selection, we first explored the robustness of VM_SCS to the initial boundary in the shape reconstruction. For better demonstration, two 2-D images both having $40 \times 40$ pixels were chosen for our analysis (Fig. 1a or b). One contains two separate somas, and the other contains two contiguous somas. Note that, in the analysis of 2-D images, VM_SCS reconstructs the object's shape by solving the variational model in the polar system, and the corresponding parameters were set to the same as that of 3-D images. This was also suitable for the following analysis of 2-D images.

In Fig. 1a, the results for the two images reconstructed using the active contour model showed strong dependence on the initial boundary (black circle). For the unsuitable initial boundary, its boundary elements converged to a fixed point (blue point, Fig. 1a), which usually results from the lacking of constraint on the direction that boundary element moves towards. This is illustrated by using the movement of a boundary element that started on the initial boundary, moved towards and reached the edge of soma, and continued to move along the edge and finally reached a fixed position, as shown with the green curved arrows (Fig. 1a). With the same images and the initial boundaries used in Fig. 1a, the shapes reconstructed using VM_SCS were almost identical (Fig. 1b), which indicated that VM_SCS have more robustness to the selection of the initial boundary. In contrast to active contour model, VM_SCS makes all the boundary elements move along their own rays (green straight arrow, Fig. 1b), in other words, the direction that each boundary element moves along is determined by polar angle and azimuth angle in sphere coordinate system, and thus VM_SCS can fix the directions of these elements.

Furthermore, we quantified the robustness of VM_SCS to the initial boundary. In the calculations, all the initial boundaries were set to circles with uniformly and randomly positioned centers across a given square area. The square was located at the center of soma with $6 \mu \mathrm{m}$ side length. The size of the initial circle radius ranged from $1.2 \mu \mathrm{m}$ to $6 \mu \mathrm{m}$. This setting can avoid some multiple somas lying in the initial boundary, according to our results that the minimum value of the soma radius is about $3.5 \mu \mathrm{m}$. For each fixed radius value, 200 simulated initial circles with different centers were generated and 

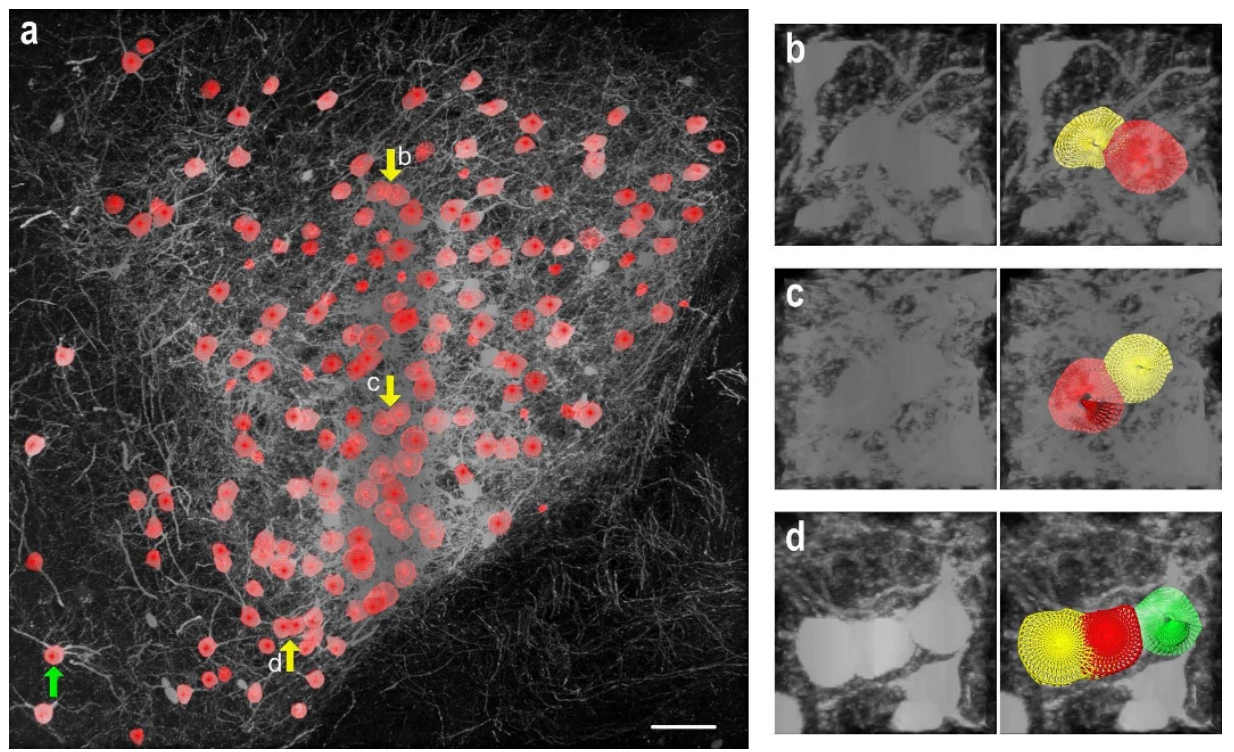

Figure $2 \mid$ Shape reconstruction for the neuronal somas with dense spatial distribution. (a) Max-intensity projection of image stacks and shape reconstruction for the neuronal somas. Some reconstructions of the closely positioned somas are given (labeled in yellow arrows in (a)) and enlarged in (b), (c) and (d) respectively. Scale bar $50 \mu \mathrm{m}$ in (a).

divided equally into two parts. Then they were analyzed using VM_SCS and active contour model respectively. To give a quantitative estimation, a 15\% or less relative error in areas between automatically and manually reconstructed shapes is considered as an accurate reconstruction. This standard is also used in our following analysis. We calculated the rate of accurate reconstruction of VM_SCS and the active contour model with respect to a fixed radius of the initial circles with different centers. VM_SCS rendered an almost unchanged accurate reconstruction rate under a wide setting of initial boundary conditions, whereas the results using active contour model were largely dependent on the initial boundary (Fig. 1c). From these results, we conclude that VM_SCS has far more robustness than active contour model in terms of the initial boundary settings.

Next we will explain why the reconstructions of active contour model were strongly dependent on the setting of initial boundary. Generally, in active contour model, gradient vector flow (GVF) and the smoothing force act simultaneously on the given initial boundary and make it evolve to the real boundary. GVF, the smoothing gradient of the boundary map, is supposed to point towards the real boundary in an ideal case, i.e. keeps high isotropy for our analysis. However, in Fig. 1d, there is an abrupt change in the normalized intensity of GVF on the blue curve boundary. This intensity inhomogeneity leaded to low isotropy of GVF in the neuronal soma, and damaged the shape reconstruction. It was illustrated by the example shown in Fig. 1e. Some GVF with the strong intensity can point towards the boundary, while others have the irregular directions (the black arrows in Fig. 1e). In this case, GVF with low isotropy made the unsuitable initial boundary (black circle) evolve to the red curves, to a part of the real boundary (green curve Fig. 1e) and finally to the given point (blue point in Fig. 1e).

Shape reconstruction for the neuronal somas with different spatial distribution. To evaluate the capability of VM_SCS in reconstructing the shape of the neuronal somas with different spatial distribution, experimental image stacks with the size of 500 $\times 500 \times 20$ volume pixels were analyzed. The neurons shown in Fig. 2a have different spatial distribution ranging from sparse to dense. It can be seen from the results that while VM_SCS is effective in reconstructing sparsely distributed neuronal somas (green arrows in Fig. 2a), it also yields high-precision shape reconstruction results for contiguous neuronal somas. As shown in
Fig. 2a (yellow arrows) and the enlarged images of Fig. 2b-d, largely contiguous somas can be accurately reconstructed. Please note that we directly ignored the somas near the edge of image stacks, and thus some bright regions in Fig. 2a were not segmented.

Furthermore, we also used simulation datasets to quantify VM_SCS for this purpose at different noise levels. Each dataset contained a pair of $5 \mu \mathrm{m}$ radius spheres with central distance ranging from $8 \mu \mathrm{m}$ to $11 \mu \mathrm{m}$. The central distance here was denoted as $d$ to show whether these spheres were closely positioned. The size of volume pixels was set to $0.5 \times 0.5 \times 0.5 \mu \mathrm{m}^{3}$, and each dataset had $60 \times 60 \times 60$ volume pixels. The datasets were generated as follows. Inside the spheres, the volume pixel's value was set to be a Poisson random number whose expectation was the sum of the signal intensity $I_{0}$ and the background $I_{b}$. Outside the spheres, the value was Poisson random number with expectation $I_{b}$. The signalto-noise rate (SNR) is defined as the ratio of $I_{0}$ to the standard deviation of $I_{0}+I_{b}$. $I_{b}$ was a fixed value of 100 for all simulation datasets. Nine typical datasets were presented in Fig. 3a. Fig. 3b and $3 \mathrm{c}$ show the statistical information of the reconstructed sphere shape from the datasets. In shape reconstruction of single sphere, for each ray that a boundary element moved along, we computed the distance between the original position (center position of the sphere) and the position that the boundary element finally reached. Then the average and variance values of these distances were calculated (Fig. $3 \mathrm{~b}$ and $3 \mathrm{c}$ ). By comparing them to the ideal reconstruction, in which the average and variance should be $5 \mu \mathrm{m}$ and $0 \mu \mathrm{m}^{2}$ respectively, we conclude that VM_SCS can reconstruct the shapes of the severely closely positioned somas at the suitable level of SNR. The reconstructions were further quantified in Fig. $3 \mathrm{~d}$ by using the previous standard that a $15 \%$ or less relative error in cubage between automatically reconstructed and real spheres. For severely closely positioned somas $(d=8 \mu \mathrm{m})$, a correct rate of $80 \%$ can be achieved if signal-to-noise was more than 2 . If signal-to-noise ratio dropped to 1.5 , the shapes of those closely positioned somas $(d=9 \mu \mathrm{m})$ can be effectively reconstructed with a correct rate of $96 \%$. From the above results, we conclude that VM_SCS can effectively reconstruct shapes of separately and closely positioned somas at low level of signal-tonoise.

Shape reconstruction of neuronal somas with different size and complicated morphology. We also used experimental datasets to 


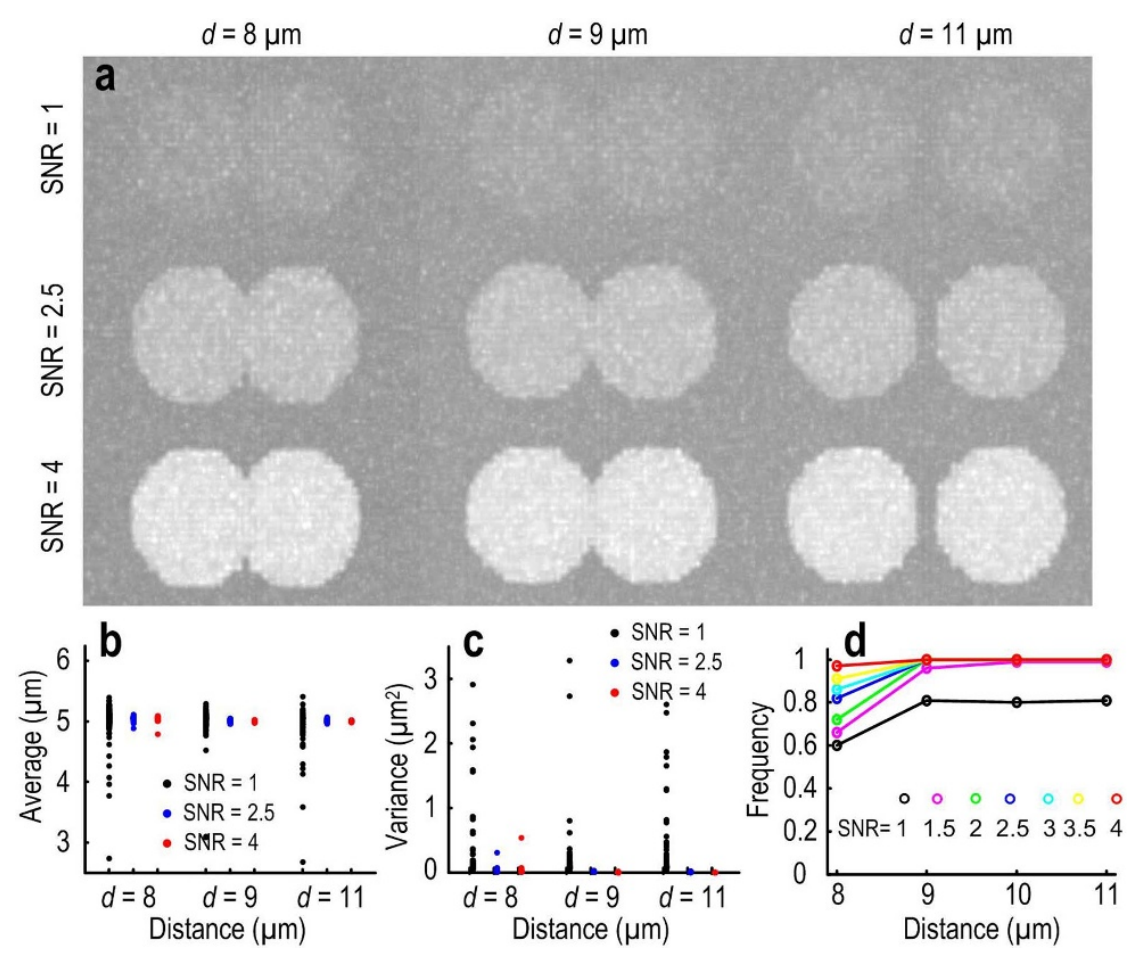

Figure 3 Reconstruction performance of VM_SCS on the simulation somas with different spatial distribution and at different level of SNR. (a) X-y projections of nine pairs of simulation spheres with fixed radius of $5 \mu \mathrm{m}$ presented. Here the alphabet of $d$ represents the distance between the center positions of a pair of spheres. (b) The average lengths of rays in shape reconstruction of single sphere are shown and denoted by a point. The length of a ray means the distance between center position of the sphere and the reconstructed boundary position on this ray. For a fixed simulation parameters $(d$, SNR), 50 pairs spheres generated and their shapes reconstructed. Correspondingly, 100 points were shown for each group of parameters ( $d$, SNR). (c) The variance of lengths of rays are shown when the distance $d$ and SNR changes. (d) The accurate reconstruction rate as a function of the distance between the central positions of a pair of spheres for different SNRs ranging from 1 to 4 .

show that VM_SCS can reconstruct the shape of the neuronal somas with different size and complicated morphology. As shown in Fig. 4a, some neurons have long and thick trucks, resulting in the complicated morphology; the difference in the size of neuronal somas obviously exists. In the shape reconstruction of these neuronal somas, VM_SCS effectively eliminated the interference from those thick dendritic trucks and achieved accurate reconstruction (labeled as red surface in Fig. 4a). We also calculated the surface area and the cubage of digitized somas (Fig. $4 \mathrm{~b}$ and c) respectively, using the protocol ${ }^{43}$. The range of the surface area and the cubage of these somas are from 437 to $2373 \mu \mathrm{m}^{2}$ and 680 to $8418 \mu \mathrm{m}^{3}$ respectively, which further verifies the large variance in the size of neuronal somas.

Comparison of reconstruction results between VM_SCS and other methods. To further examine efficiency of VM_SCS, we also compared the reconstruction performance of active contour model, rayburst sampling and VM_SCS (Fig. 5). The experimental image has $1200 \times 400$ pixels with a pixel size of $1.2 \times 1.2 \mu \mathrm{m}^{2}$. In this image, many somas are closely positioned, and some of them are connected to the thick dendritic trucks, which is rather challenging for reconstruction. Before testing these methods, the positions of somas were manually given (black points in Fig. 5a-c), and the results were shown by red curves. In Fig. 5a, the shape of somas reconstructed using VM_SCS agreed well with those provided by the manual. By using the previous standard, among 129 somas there was only one soma whose shape can't be reconstructed. However, rayburst sampling method only obtained the boundary details of separately positioned somas, and cannot reconstruct the shapes of the neighboring somas contiguous to each other, as shown in the enlargement of the squares (Fig. 5b). 73 somas were successfully reconstructed (the correct rate of 57\%) using rayburst sampling. When active contour model was used (Fig. 5c), due to the low isotropy of GVF in a neuronal soma caused by the disturbance of other somas or thick dendrite trucks, some reconstructions were points or open curves, as pointed out by the arrows in Fig. 5 c. In this reconstruction, the correct rate of $77 \%$ was achieved. Obviously, we conclude that VM_SCS is more suitable for shape reconstruction for somas in complex neural images.

\section{Discussion}

In this paper, we have proposed the VM_SCS method to reconstruct the morphology of neuronal somas. In complicated image stacks of neurons, VM_SCS method can effectively reconstruct the shape of the clustered neuronal somas, and eliminate the interference from thick dendritic truck during reconstruction. We also show that VM_SCS is highly robust to the size variation of neuronal somas and the initial boundary in the reconstruction.

Like the active contour model ${ }^{47,48}$, our VM_SCS uses a combination of the gradient signal and the smoothness force to drive the evolution of boundary elements to convergence. These convergent positions of boundary elements form the object's shape. It is clear, when some boundary elements evolve to the common boundary between two somas contiguous to each other or a soma and a thick dendritic truck, the gradient signal is weak, and the smoothness force is dominate, making these boundary elements stay at the common boundary, i.e., the correct positions. Based on this, our VM_SCS can reconstruct the shape of the clustered somas (Fig. 2,3,) and eliminate the interference from the thick dendritic truck (Fig. 4).

Different from active contour model ${ }^{47,48}$, our VM_SCS shows high robustness to the setting of the initial boundary. In active contour model $^{48}$, the concept of gradient vector flow (GVF) is introduced to reconstruct the object's boundary. For applications with simple soma shape, the GVF diffuses in an isotropic manner, which keeps the 

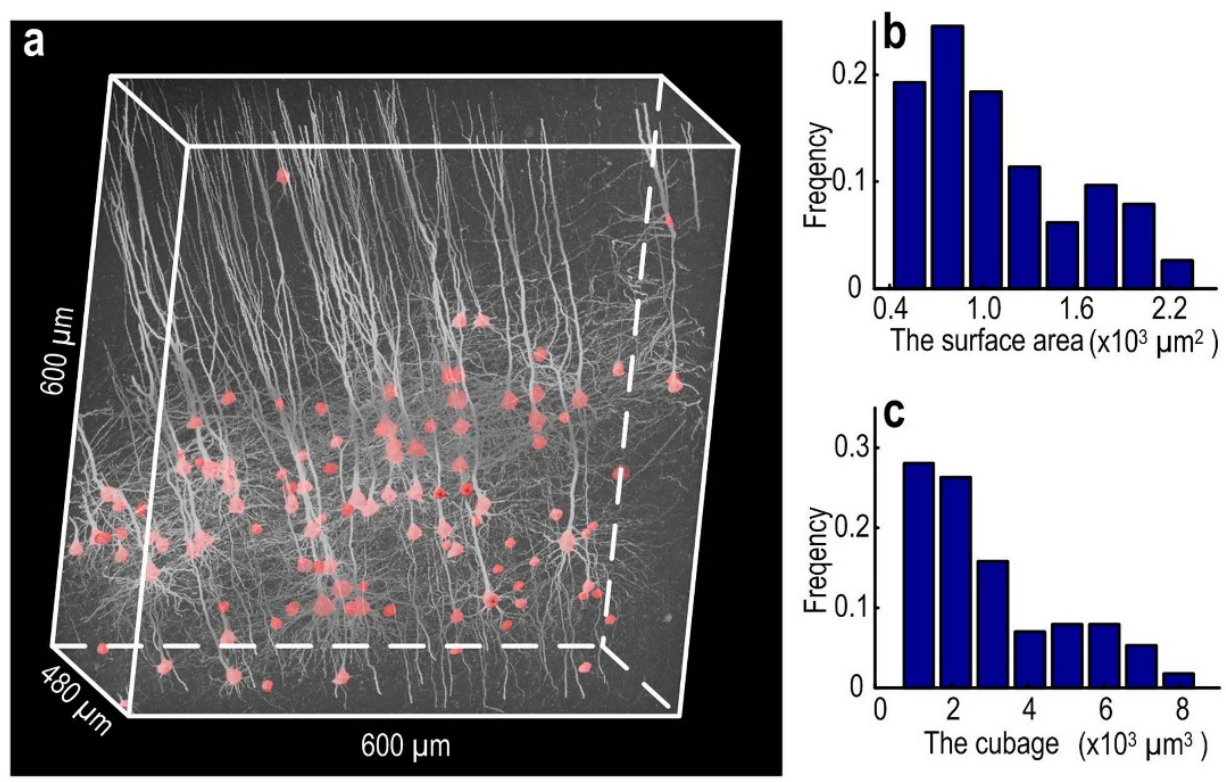

Figure $4 \mid$ Shape reconstruction for the somas of the neurons with different size and complicated morphology. (a) Neurons with complicated dendritic trucks and the reconstructed soma shape (red). The density distribution of the surface area (b) and the cubage (c) of these reconstructed neuronal somas show the size diversity.
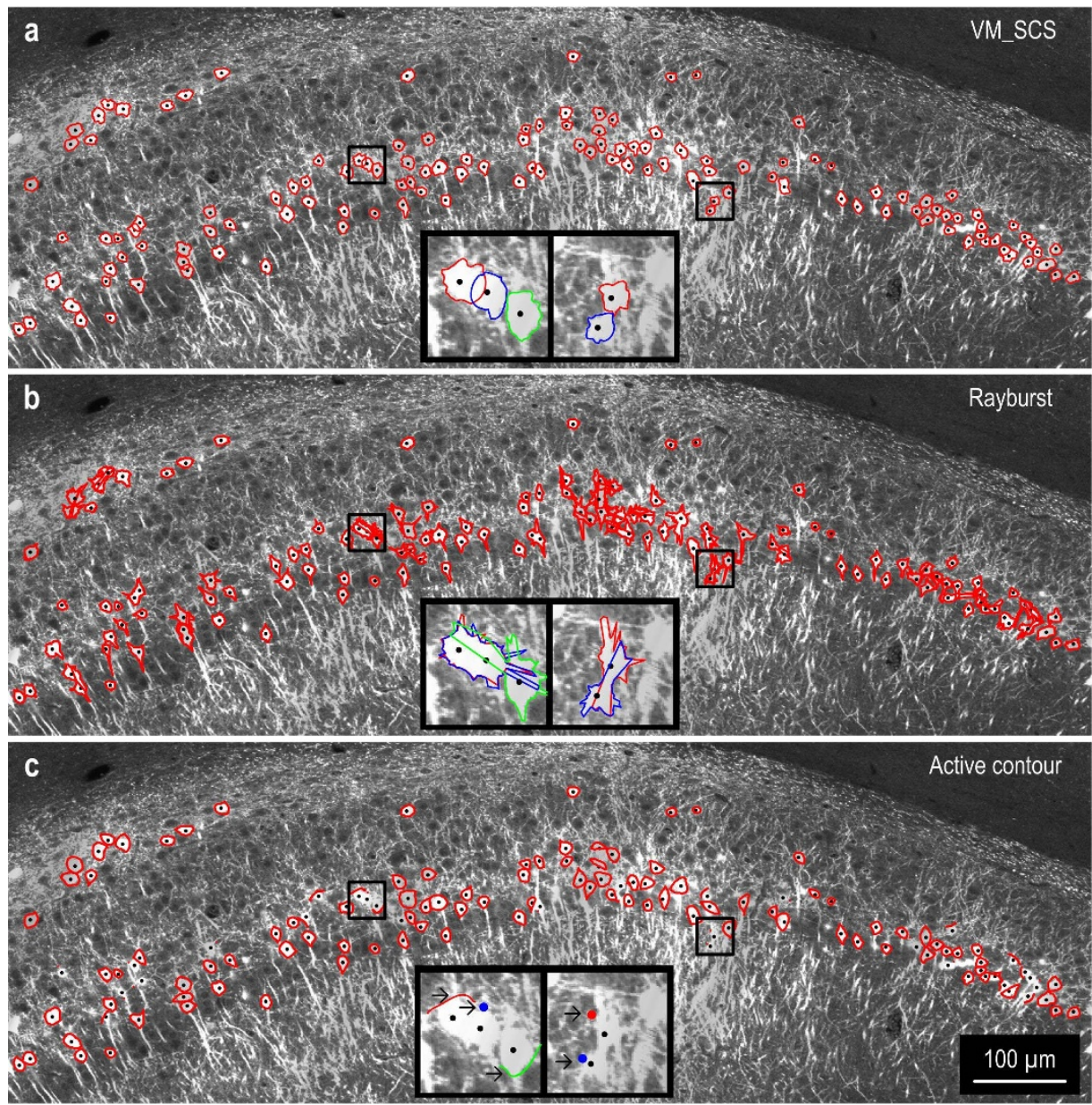

Figure 5 $\mid$ A comparison of three shape reconstruction methods on the experimental data. (a)The shapes reconstructed using VM_SCS under the condition of a given soma position, denoted by black points. (b) The shapes reconstructed using rayburst sampling that a rays were casted from a soma position (black points) and the threshold method were used to compute the length of the ray. (c) The shapes reconstructed using active contour model. All the initial boundaries were circles centered on their soma positions (black points) and whose radiuses were set to $5 \mu \mathrm{m}$ for best results. (a) - (c) The results for two subareas (small black squares) selected for comparison, and further enlarged (big black squares). Note that, in the enlargement, the reconstructed shapes of different somas were distinguished using different colors, and the points or open curves that the arrows pointed towards still denote the reconstructed results. 

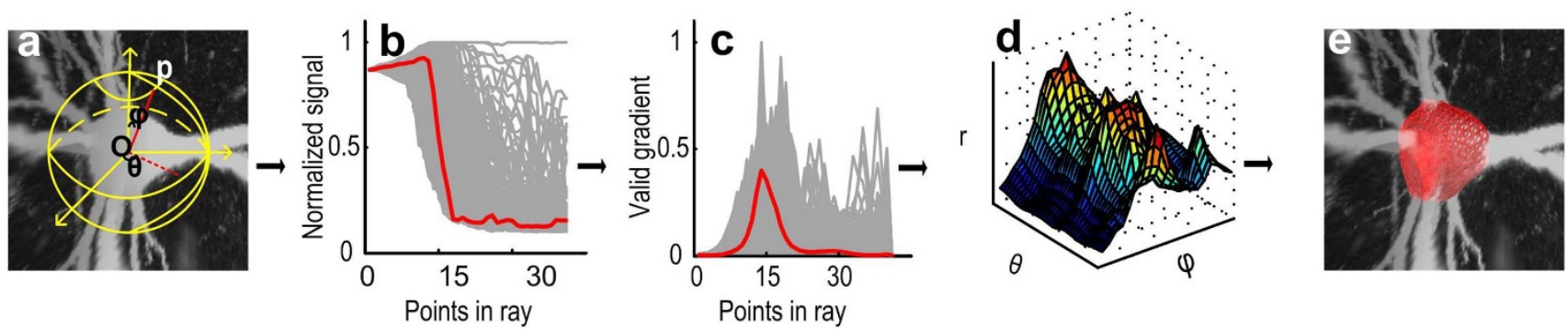

Figure 6 | Scheme for the shape reconstruction routine of VM_SCS. (a) The sphere coordinate system with origin position $O$ used to describe the signal from the image stacks. (b) The normalized signals in rays obtained via data sampling in sphere coordinate system. For each ray, it was equally divided into 39 parts, and its signal is generated by averaging the signals of each dividing point (including endpoints) and its 6 neighbors. (c) The valid gradient signal of each ray was calculated from the curve in (b) with respect to the same ray, and generated by setting the negative value of the smoothing gradient signal to its converse value and setting the positive value to zero. The red curves in (b) and (c), corresponding to the red ray OP in (a), served as an example for illustrating how to obtain the gradient signal. (d) By iteratively computing the equation (7), which can minimize the energy function, the curve surface in sphere coordinate system was obtained from the signals in (c). The third component value of a point in the curve surface is the distance between the computed boundary position to origin position $O$ in the ray determined by the others components i.e., polar angle and azimuth angle. (e) Soma shape formed by the computed boundary positions in the rays.

boundary elements move along their correct evolution directions. However, for applications with complicated neuronal morphology as described in this paper, this isotropic diffusion property of GVF might be broken for some initial boundaries, which leads to failed identification, as shown in the active contour model in Fig. 1 and Fig. 5c. While in VM_SCS method, the boundary element moves along its ray direction with the help of the sphere coordinate system, and naturally keeps the isotropic diffusion property during evolution. This design contributes to high robustness of VM_SCS to the setting of initial boundary (Fig. 1 and Fig. 5a).

Rayburst sampling ${ }^{43-46}$ methods reconstruct the object shape according to the following steps: casting rays from the given point; computing the length the rays by a comparison of the signal intensity and a given threshold; using the length of rays for shape recovery. Due to a wide range of the number of sampling points in each ray, ray burst sampling can reconstruct the object's shape on different scales $^{43-46}$. However, for clustered neuron in dense neural circuits, the signal intensity in the common boundary of the contiguous somas is stable, and does not experience a decrease below the threshold. So, rayburst sampling method cannot find the boundary of the contiguous somas (Fig. 5). VM_SCS obtains the optimal surface by minimizing the designed variational model (1st paragraph of Methods), shares the merits of active contour model (2nd paragraph of Discussion), and thus overcomes the inability of rayburst sampling in reconstructing the shape of clustered objects.

The design of VM_SCS that the boundary element moves along its ray direction is based on the underlying fact that most neuronal somas have star-convex structure and can be presented in sphere coordinate system without information loss. This design contributes to the competitive results when using VM_SCS to analyze the complicated images (Fig. 1 and Fig. 5); meanwhile, this design also reduces the applied ranges of VM_SCS, namely, VM_SCS may be unable to completely reconstruct the non-star-convex shape. However, the case that a soma has non-star-convex shape is very rare according to our experience with thy-1 transgenic mouse; no exception is met across different brain areas including the cortex, the hippocampus, and epencephala.

The robustness of VM_SCS against partial volume effects may be an important issue in shape reconstruction. However, in the shape reconstruction of cells field, due to the fact that molecular labeling technique characterized by specificity make partial volume effects negligible in imaging cells ${ }^{12,13,49}$, few algorithms have taken the issue of robustness against partial volume effects into account ${ }^{23-46}$. Additionally, the issue involved in partial volume effects usually appears in the MRI and PET images ${ }^{50}$. Our VM_SCS aims at shape reconstruction of somas or other cells at present, and may not be directly applicable to MRI images because VM_SCS can't completely reconstruct some non-convex shapes. So, before applying VM_SCS to analyze MRI or PET images, the issue involved in partial volume effect should be considered in depth.

For the high-throughput image stacks of neurons, the computing time of VM_SCS should be a matter of concern. In VM_SCS, sampling data in sphere coordinate system is necessary and will bring redundant data. However, compared to active contour model ${ }^{48}$, VM_SCS fixes the evolution direction of boundary element and has a much faster convergence. Generally, using VM_SCS to reconstruct a neuronal soma takes about one second in an Inter(R)Xeon(R)CPU $3.46 \mathrm{GHz}$ computing platform. This speed should be similar to its in active contour mode ${ }^{48}$ according to our estimation.

It should be noted that a rough estimation of position of neuronal soma is necessary before using VM_SCS for shape reconstruction. For neurons with complicated morphology, we have proposed NeuroGPS method ${ }^{51}$ to automatically locate the neurons across different brain areas. We will combine VM_SCS with NeuroGPS method to automatically extract some features of neuronal soma including position, surface area, cubage, and so on. With the advantages of VM_SCS, we firmly believe that VM_SCS can also be applied to the reconstruction of morphology of complicated neuritis and spines, and should be a powerful computational tool for the digitalization of neurons and drawing neural circuits.

\section{Methods}

Variational model in spherical coordinate system (VM_SCS) for shape reconstruction. In a spherical coordinate system with a fixed origin $O$, the position of a point $P$ can be specified by the parameters $r, \varphi, \theta$. Here, $r, \varphi, \theta$ denote the distance of the point $P$ and the point $O$, polar angle, and azimuth angle respectively (See Fig. 6a). The morphology of neuronal soma can be modeled by the function $r(\varphi, \theta)$ in the spherical coordinate system. The task of shape reconstruction of neuronal soma is transferred to estimating the function $r(\varphi, \theta)$ from the image stacks. To characterize the function $r(\varphi, \theta)$, we assumed that the morphology of neuronal soma is smoothness to some extent, corresponding to smoothness of the function $r(\varphi, \theta)$, i.e., a sharp change of $r$ is impossible when $\varphi, \theta$ vary slowly, and that the intensity of differential signal on the boundary of neuronal soma is stronger than that in the inside and outside of soma. Based on the above assumptions, by minimizing the following energy function

$$
E=\int_{0}^{2 \pi} \int_{0}^{\pi}\left\{\frac{\alpha}{2}\left(\alpha_{1}\left(\frac{\partial r}{\partial \varphi}\right)^{2}+\alpha_{2}\left(\frac{\partial r}{\partial \theta}\right)^{2}\right)-\beta E_{e x t}(r(\varphi, \theta))\right\} d \varphi d \theta
$$

we can obtain the function $r(\varphi, \theta)$. Here the parameter $\alpha$ is the tradeoff between the smoothness of the function $r(\varphi, \theta)$ and the loss of reconstructed details of neuronal soma. A big value of $\alpha$ will increase the smoothness of the function $r(\varphi, \theta)$ and damage the reconstruction of the details of neuronal soma. Conversely, accurate reconstruction of the details is built on a sacrifice for the smoothness of the function $r(\varphi, \theta)$. The parameters $\alpha_{1}, \alpha_{2}, \beta$ are introduced to simplify the numerical calculation of $r(\varphi, \theta) . E_{\text {ext }}(r(\varphi, \theta))$ is a function with respect to $r(\varphi, \theta)$, and can be written as 


$$
E_{\text {ext }}(r(\varphi, \theta))=\int \sigma^{2} g(s) \exp \left(\frac{-\|s-r\|^{2}}{2 \sigma^{2}}\right) d s
$$

where the function $g(s)$ is used to describe the intensity of the differential signal with respect to the position $s$ in the ray determined by the parameters $\varphi, \theta$. The detailed calculation of $g(s)$ is in the section (see the entire processing of shape reconstruction, Step 2). Note that maximizing the function $E_{\text {ext }}(r(\varphi, \theta))$ can be considered as finding the peak value of the function $g(s)$. Generally, the position $s$ corresponding to the peak value of $g(s)$ lies on the boundary of neuronal soma.

The numerical solution of VM_SCS. To minimize the energy function (1), we introduce the procedure that is similar to the active contour method ${ }^{48}$. According to the well-known fact, if the curve surface $r(\varphi, \theta)$ is the optimal solution that minimizes the energy function (1), it must satisfy the Euler equation

$$
\frac{\alpha}{2}\left(\alpha_{1} \frac{\partial^{2} r}{\partial \varphi^{2}}+\alpha_{2} \frac{\partial^{2} r}{\partial \theta^{2}}\right)-\beta \nabla E_{e x t}=0
$$

To solve the equation (3), we treat $r(\varphi, \theta)$ as a function of time $t$, polar angle $\varphi$, and azimuth angle $\theta$, and establish the new equation as

$$
\frac{\partial r(\varphi, \theta, t)}{\partial t}=\frac{\alpha}{2}\left(\alpha_{1} \frac{\partial^{2} r(\varphi, \theta, t)}{\partial \varphi^{2}}+\alpha_{2} \frac{\partial^{2} r(\varphi, \theta, t)}{\partial \theta^{2}}\right)-\beta \nabla E_{e x t}
$$

when $r(\varphi, \theta, t)$ stabilizes, i.e., the right hand side of (4) approaches zeros, a solution of (3) can be achieved. By substituting equation (2) into equation (4), equation (4) can be rewritten as

$$
\begin{aligned}
\frac{\partial r(\varphi, \theta, t)}{\partial t} & =\alpha\left(\alpha_{1} \frac{\partial^{2} r(\varphi, \theta, t)}{\partial \varphi^{2}}+\alpha_{2} \frac{\partial^{2} r(\varphi, \theta, t)}{\partial \theta^{2}}\right) \\
& -\beta \int g(s) \exp \left(\frac{-\|s-r\|^{2}}{2 \sigma^{2}}\right)(s-r) d s
\end{aligned}
$$

To obtain the stable $r(\varphi, \theta, t)$, we discretize the equation (5)

$$
\begin{aligned}
& \frac{r_{i, j, k+1}-r_{i, j, k}}{\Delta t} \\
& =\alpha\left(\frac{r_{i+1, j, k}-r_{i, j, k}}{\Delta \varphi / \alpha_{1}}-\frac{r_{i, j, k}-r_{i-1, j, k}}{\Delta \varphi / \alpha_{1}}+\frac{r_{i, j+1, k}-r_{i, j, k}}{\Delta \theta / \alpha_{2}}-\frac{r_{i, j, k}-r_{i, j-1, k}}{\Delta \theta / \alpha_{2}}\right) \\
& -\beta \sum_{m=1}^{M} g\left(s_{m, i, j}\right) \exp \left(\frac{-\left\|s_{m, i, j}-r_{i, j, k}\right\|^{2}}{2 \sigma^{2}}\right)\left(s_{m, i, j}-r_{i, j, k}\right)
\end{aligned}
$$

where $r_{i, j, k}$ is an abbreviation of $r\left(\varphi_{i}, \theta_{j}, t_{k}\right)$ and recognized as the function of the boundary element $\left(\varphi_{i}, \theta_{j}\right)$, and $s_{m, i j}$ is the sampling point in the ray determined by the parameters $\varphi_{i}, \theta_{j}$. Both $\Delta \varphi / \alpha_{1}$ and $\Delta \theta / \alpha_{2}$ are set to one for simplifying equation (6), we can obtain

$$
\begin{aligned}
r_{i, j, k+1}= & r_{i, j, k}-\Delta t \alpha\left(4 r_{i, j, k}-r_{i, j+1, k}-r_{i, j-1, k}-r_{i-1, j, k}-r_{i+1, j, k}\right) \\
& -\Delta t \beta \sum_{m=1}^{M} g\left(s_{m, i, j}\right) \exp \left(\frac{-\left\|s_{m, i, j}-r_{i, j, k}\right\|^{2}}{2 \sigma^{2}}\right)\left(s_{m, i, j}-r_{i, j, k}\right)
\end{aligned}
$$

By iteratively computing $r_{i, j, k+1}$ until convergence, the numerical solution of $r(\varphi, \theta)$ can be achieved.

The entire processing of shape reconstruction. This section contains three steps. In step 1, we described signal sampling in the sphere coordinate system. From the sampled signals (Fig. 6b), we computed the valid nonnegative gradient signals (Fig. 6c) denoted as the function $g$ in Eq. (7), which was illustrated in step 2. After obtaining the valid gradient signal, we iteratively computed Eq. (7) until convergence, and the surface reconstruction can be achieved (Fig. $6 \mathrm{~d}$ and e), which was illustrated in step 3. Detailed description of these steps is as follows.

Step 1. Signal sampling in the sphere coordinate system. In this section, the process of signal sampling in the sphere coordinate system with origin $O$ is introduced. We discrete polar angle and azimuth angle respectively

$$
\varphi_{i}=i / N_{1} \pi \quad\left(i=1,2, \cdots, N_{1}\right), \theta_{j}=2 j / N_{2} \pi \quad\left(j=1,2, \cdots, N_{2}\right)
$$

with $N_{1}$ and $N_{2}$ being setting to 20 and 40 respectively. The position $s_{m, i, j}$ in the ray with polar angle $\varphi_{i}$ and azimuth angle $\theta_{j}$ can be represented as

$$
s_{m, i, j}=o+r_{m}\left(\cos \varphi_{i} \cos \theta_{j}, \cos \varphi_{i} \sin \theta_{j}, \sin \varphi_{i}\right)^{T}
$$

where $r_{m}$ with the sampling step of half of a voxel denotes the distance of the position $s_{m, i, j}$, and $T$ denotes the transpose operation. The signal with respect to the position $s_{m, i, j}$ is computed by

$$
v\left(s_{m, i, j}\right)=\frac{\sum_{s \in U\left(\left[s_{m, i, j}\right]\right)} \exp \left(\frac{-\left\|s-s_{m, i, j}\right\|^{2}}{2}\right) v(s)}{\sum_{s \in U\left(\left[s_{m, i, j}\right]\right)} \exp \left(\frac{-\left\|s-s_{m, i, j}\right\|^{2}}{2}\right)}
$$

Here, [] represents rounding the element to its nearest integer and $U(s)$ denotes the set including the position $s$ and its 6-connected neighborhood, $v(s)$ is the signal value of position $s$ from the image stacks. Each ray was uniformly divided into $40 \mathrm{sub}$ intervals and its corresponding signal has been shown in Fig. 6b. The length of rays should be more than the biggest value of the radius of neuronal soma.

Step 2. Calculation of the valid gradient signal in rays. For the given ray, the corresponding negative gradient signal was calculated from the signals $v\left(s_{m}\right)$ with $m$ $=1,2, \ldots, \mathrm{M}$. The procedure is as follows

a) Calculate the differential signal: $\nabla v\left(s_{m}\right)=v\left(s_{m+1}\right)-v\left(s_{m}\right), m=1,2, \ldots, \mathrm{M}$.

b) Using linear interpolation to calculate the value of $\nabla v\left(s_{m}\right), m=1,1.5,2, \ldots, \mathrm{M}-$ $1.5, \mathrm{M}-1$.

c) Using the weighted averaging method to smooth the $\operatorname{signal} \nabla v\left(s_{m}\right)$

$\nabla v\left(s_{m}\right) \leftarrow \frac{\left|\nabla v\left(s_{m}\right)\right| \nabla v\left(s_{m}\right)+2 \nabla v\left(s_{m-0.5}\right)+2 \nabla v\left(s_{m+0.5}\right)}{\left|\nabla v\left(s_{m}\right)\right|+4}, m=1.5,2,2.5, \cdots, M-1.5$

$\nabla v\left(s_{m}\right)$ is repeatedly computed until all small peaks in $\nabla v\left(s_{m}\right)$ disappear.

d) Calculate the negative gradient signal from $\nabla v\left(s_{m}\right)$

$$
g\left(s_{m}\right)=-\min \left(\nabla v\left(s_{m}\right), 0\right), \quad m=1,2, \cdots, M-1
$$

By these above operations, $g(s)$ is finally generated and regarded as the valid gradient signal (Fig. 6c). Considering that in our experimental image stacks, the size of volume pixels is $1.2 \times 1.2 \times 2.4 \mu^{3}$, and sampling step is half of a volume pixels, we set $\mathrm{M}$ to 40 for all analysis, which is suitable for our experimental datasets.

The smoothing operation in $c$ ) can achieve that the big and small value of the signal $\nabla v\left(s_{m m}\right)$ changes slowly and sharply respectively. This also means that we can keep the big peaks usually around the boundary of object shape, and discard the small peaks in the signal using a broad range of the number of iterative smoothness computation. In our dataset analysis, the iterative computation was carried out 100 times.

In $d$ ), we select the negative gradient signal as the valid signal based on the following facts: the signal intensity of intra-soma is more than that of extra-soma; the signal in sphere coordinate system was sampled from the intra-soma to the extrasoma. We can conclude that the differential signal around the boundary of neuronal soma should be negative, which indicates that the nonnegative differential signal are useless for the evolution of boundary element and its value can be set to zeroes. In addition, considering that the function (2) should be maximized for shape reconstruction, and that the position around the boundary of neuronal soma should approach the position of negative peak value of $v\left(s_{m}\right)$, the negative value of $v\left(s_{m}\right)$ was set to its opposite value.

Step 3. Surface reconstruction. For the surface reconstruction, we repeatedly calculate the equation (7) until all the boundary elements $\left(\varphi_{i}, \theta_{j}\right)$ converge to the fixed positions $r_{i, j}$. These fixed positions formed the surface function $r(\varphi, \theta)$ (see Fig. $6 \mathrm{~d}$ and e). In equation (7), the parameter $\beta$ was set to

$$
\beta=\frac{\beta_{0}}{\sum_{m=1}^{M} g\left(s_{m}\right) \exp \left(\frac{-\left\|s_{m}-r_{i, j, k}\right\|^{2}}{2 \sigma^{2}}\right)}
$$

With $\sigma=0.5$, and $\Delta t$ is set to one. The setting of unknown parameters $\alpha$ and $\beta_{0}$ was divided into two stages. In the first stage, to assure that the majority of boundary elements can reach the boundary of neuronal soma, the force of the smoothness in controlling evolution of boundary element should be reduced, and $\alpha$ and $\beta_{0}$ were set to 0.2 and 0.8 respectively. In the second stage, for the minority of boundary elements that cannot achieve the boundary due to the small values of the nonnegative gradient signal, we enforce the smoothness for their evolution. The parameter $\alpha$ keeps unchanged and $\beta_{0}$ is set to 0 .

Brain tissue preparation. All experiments were performed in accordance with the guidelines of the Experimental Animal Ethics Committee at Huazhong University of Science and Technology. The THY1-EGFP-M line transgenic fluorescence mouse (adult (P41) male) was deeply anaesthetized, and transcardially perfused with $50 \mathrm{~mL}$ phosphate buffered saline (PBS) $(0.1 \mathrm{M})$ and $200 \mathrm{~mL} 4 \%$ paraformaldehyde (PFA). The whole brain was brought out, post-fixed in $4 \%$ PFA for 12 hours at $4 . \mathrm{C}$, rinsed in PBS thrice (for $2 \mathrm{~h}, 2 \mathrm{~h}$, and $12 \mathrm{~h}$ respectively), and then infiltrated in a graded series of GMA. Finally, the brain was embedded in gelatin capsules and polymerized for $60 \mathrm{~h}$ at $60^{\circ} \mathrm{C}$. A detailed description of the sampling preparation can be found in Ref 52.

Imaging system. The whole brain was sliced and imaged using two-photon fluorescence micro-optical sectioning tomography system $(2 p-f M O S T)^{52}$ that consists of dispersion compensation, fast scanning, microscope, and motion and sectioning 
modules. The experimental lateral and axial spatial resolution of $2 p-f M O S T$ are about $0.45 \mu \mathrm{m}$ and $1.8 \mu \mathrm{m}$ respectively, and the size of volume pixel is down sampled to be $1.2 \times 1.2 \times 2.4 \mu^{3}$. These values of the parameters are sufficiently high to resolve the soma shape. The datasets were generated as follows. The image tiles were mosaicked to form a whole section. In each section, the inhomogeneous illustration patterns were removed using similar procedures proposed previously $y^{53}$. An entire brain dataset contained about 6000 sections. A few parts selected from the entire dataset were used for our analysis.

1. Peng, H., Ruan, Z., Long, F., Simpson, J. H. \& Myers, E. W. V3D enables real-time $3 \mathrm{D}$ visualization and quantitative analysis of large-scale biological image data sets. Nat. Biotechnol. 28, 348-353 (2010).

2. Helmstaedter, M. \& Mitra, P. P. Computational methods and challenges for largescale circuit mapping. Curr. Opin. Neurobiol. 22, 162-169 (2012).

3. Lichtman, J. W. \& Denk, W. The big and the small: challenges of imaging the brain's circuits. Science 334, 618-623 (2011).

4. Lu, J. Neuronal tracing for connectomic studies. Neuroinformatics 9, 159-166 (2011).

5. Senft, S. L. A brief history of neuronal reconstruction. Neuroinformatics 9,119-128 (2011).

6. Egger, R., Narayanan, R. T., Helmstaedter, M., deKock, C. P. \& Oberlaender, M. 3D Reconstruction and Standardization of the Rat Vibrissal Cortex for Precise Registration of Single Neuron Morphology. PLoS Comput. Boil. 8, e1002837 (2012).

7. Binzegger, T., Douglas, R. J. \& Martin, K. A. A quantitative map of the circuit of cat primary visual cortex. J. Neurosci. 24, 8441-8453 (2004).

8. Stepanyants, A. et al. Local potential connectivity in cat primary visual cortex. Cereb. Cortex 18, 13-28 (2008)

9. Oberlaender, M. et al. Cell type-specific three-dimensional structure of thalamocortical circuits in a column of rat vibrissal cortex. Cereb. Cortex $\mathbf{2 2}$ 2375-2391 (2010)

10. Lubke, J., Roth, A., Feldmeyer, D. \& Sakmann, B. Morphometric analysis of the columnar innervation domain of neurons connecting layer 4 and layer $2 / 3$ of juvenile rat barrel cortex. Cereb. Cortex 13, 1051-1063 (2003).

11. Meyer, H. S. et al. Cell type-specific thalamic innervation in a column of rat vibrissal cortex. Cereb. Cortex 20, 2287-2303 (2010).

12. Feng, G. et al. Imaging neuronal subsets in transgenic mice expressing multiple spectral variants of GFP. Neuron 28, 41-51 (2000).

13. Zhang, B. et al. Modified Golgi-Cox method for micrometer scale sectioning of the whole mouse brain. J. Neurosci. Meth. 197, 1-5 (2011).

14. Li, A. et al. Micro-optical sectioning tomography to obtain a high-resolution atlas of the mouse brain. Science 330, 1404-1408 (2010).

15. Gong, H. et al. Continuously tracing brain-wide long-distance axonal projections in mice at a one-micron voxel resolution. NeuroImage 74, 87-98 (2013).

16. Qi, X. et al. Improved detectability of neuronal connectivity on mechanical sectioning setup by using confocal detection. J. Biomed Opt. 18, 050506-050506 (2013).

17. Qu, J., Liu, L., Shao, Y., Niu, H. \& Gao, B. Z. Recent progress in multifocal multiphoton microscopy. J. Innov. Opt. Heal. Sci. 5, 1250018 (2012).

18. Ragan, T. et al. Serial two-photon tomography for automated ex vivo mouse brain imaging. Nat. Methods 9, 255-258 (2013).

19. Chung, K. et al. Structural and molecular interrogation of intact biological systems. Nature 497, 332-337 (2013).

20. Silvestri, L., Bria, A., Sacconi, L., Iannello, G. \& Pavone, F. Confocal light sheet microscopy: micron-scale neuroanatomy of the entire mouse brain. Opt. Express 20, 20582-20598 (2012).

21. Osten, P. \& Margrie, T. W. Mapping brain circuitry with a light microscope. Nat. Methods 10, 515-523 (2013).

22. Silvestri, L., Mascaro, A. A., Lotti, J., Sacconi, L. \& Pavone, F. Advanced optical techniques to explore brain structure and function. J. Innov. Opt. Heal. Sci. 6 , 12300012 (2013)

23. Malpica, N. et al. Applying watershed algorithms to the segmentation of clustered nuclei. Cytometry 28, 289-297 (1997)

24. Wahlby, C., Lindblad, J., Vondrus, M., Bengtsson, E. \& Bjorkesten, L. Algorithms for cytoplasm segmentation of fluorescence labelled cells. Anal. Cell. Pathol. 24, 101-111 (2002).

25. Lin, G. et al. Hierarchical, model-based merging of multiple fragments for improved three-dimensional segmentation of nuclei. Cytom. Part A 63, 20-33 (2005)

26. Fenistein, D., Lenseigne, B., Christophe, T., Brodin, P. \& Genovesio, A. A fast, fully automated cell segmentation algorithm for high-throughput and high-content screening. Cytom. Part A 73, 958-964 (2008).

27. Li, F., Zhou, X., Ma, J. \& Wong, S. T. An automated feedback system with the hybrid model of scoring and classification for solving over-segmentation problems in RNAi high content screening. J. Microsc. 226, 121-132 (2007)

28. Wahlby, C., Sintorn, I., Erlandsson, F., Borgefors, G. \& Bengtsson, E. Combining intensity, edge and shape information for 2D and 3D segmentation of cell nuclei in tissue sections. J. Microsc. 215, 67-76 (2004).

29. Huang, Y. et al. A computational framework for studying neuron morphology from in vitro high content neuron-based screening. J. Neurosci. Meth. 190 299-309 (2013).
30. Dufour, A. et al. Segmenting and tracking fluorescent cells in dynamic 3-D microscopy with coupled active surfaces. IEEE Trans. Image Process. 14, 1396-1410 (2005).

31. Yu, W., Lee, H. K., Hariharan, S., Bu, W. \& Ahmed, S. Quantitative neurite outgrowth measurement based on image segmentation with topological dependence. Cytom. Part A 75, 289-297 (2009).

32. Qi, X., Xing, F., Foran, D. J. \& Yang, L. Robust segmentation of overlapping cells in histopathology specimens using parallel seed detection and repulsive level set. IEEE Trans. Biomed. Eng. 59, 754-765 (2013).

33. Cheng, J. \& Rajapakse, J. C. Segmentation of clustered nuclei with shape markers and marking function. IEEE Trans. Biomed. Eng. 56, 741-748 (2009).

34. Yan, P., Zhou, X., Shah, M. \& Wong, S. T. Automatic segmentation of highthroughput RNAi fluorescent cellular images. IEEE Trans. Inf. Technol. Biomed. 12, 109-117 (2008)

35. Li, G. et al. 3D cell nuclei segmentation based on gradient flow tracking. BMC Cell Biol. 8, 40 (2007).

36. Kothari, S., Chaudry, Q. \& Wang, M. D. Automated cell counting and cluster segmentation using concavity detection and ellipse fitting techniques. In Proc IEEE Int. Symp. Biomed. Imag. 795-798 (2009).

37. Zhang, C., Sun, C. \& Pham, T. Segmentation of clustered nuclei based on concave curve expansion. J. Microsc. 251, 57-67 (2013).

38. Yu, W., Lee, H. K., Hariharan, S., Bu, W. \& Ahmed, S. Evolving generalized Voronoi diagrams for accurate cellular image segmentation. Cytom. Part A 77, 379-386 (2010).

39. Jones, T. R., Carpenter, A. \& Golland, P. Voronoi-based segmentation of cells on image manifolds. Comput. Vis. Biomed. Image Appl. 3765, 535-543 (2005).

40. Faustino, G. M., Gattass, M., Rehen, S. \& DeLucena, C. Automatic embryonic stem cells detection and counting method in fluorescence microscopy images. In Proc. IEEE Int. Symp. Biomed. Imag. 799-802 (2009).

41. Yang, L., Tuzel, O., Meer, P. \& Foran, D. J. Automatic image analysis of histopathology specimens using concave vertex graph. In Proc. Int. Conf. Med. Image Comput. Comput. Assist. Intervent. 5241, 833-841 (2008).

42. Chen, C., Li, H., Zhou, X. \& Wong, S. Constraint factor graph cut-based active contour method for automated cellular image segmentation in RNAi screening. J. Microsc. 230, 177-191 (2008)

43. Rodriguez, A., Ehlenberger, D. B., Hof, P. R. \& Wearne, S. L. Rayburst sampling, an algorithm for automated three-dimensional shape analysis from laser scanning microscopy images. Nat. Protoc. 1, 2152-2161 (2006).

44. Wearne, S. et al. New techniques for imaging, digitization and analysis of threedimensional neural morphology on multiple scales. Neuroscience 136, 661-680 (2005).

45. Yan, C. et al. Automated and Accurate Detection of Soma Location and Surface Morphology in Large-Scale 3D Neuron Images. PloS One 8, e62579 (2013).

46. Li, Q. \& Deng, Z. A Surface-Based 3-D Dendritic Spine Detection Approach from Confocal Microscopy Images. IEEE Trans. Image Process. 21, 1223-1230 (2012).

47. Kass, M., Witkin, A. \& Terzopoulos, D. Snakes: Active contour models. Int. J. Comput. Vision 1, 321-331 (1988).

48. Xu, C. \& Prince, J. L. Snakes, shapes, and gradient vector flow. IEEE Trans. Image Process. 7, 359-369 (1998).

49. Patterson, G. H. \& Lippincott-Schwartz, J. A Photoactivatable GFP for selective photolabeling of proteins and cells. Science, 297, 1873-1877 (2002).

50. Rousset, O. G., Ma, Y. \& Evans, A. C. Correction for partial volume effects in PET: principle and validation. J. Nucl. Med. 39, 904-911 (1998).

51. Quan, T. et al. NeuroGPS: automated localization of neurons for brain circuits using L1 minimization model. Sci. Rep. 3, 1414 (2013).

52. Zheng, T. et al. Visualization of brain circuits using two-photon fluorescence micro-optical sectioning tomography. Opt. Express 21, 9839-9850 (2013).

53. Ding, W. et al. Automatic macroscopic density artifact removal in a Nissl-stained microscopic atlas of whole mouse brain. J. Microsc. 251, 168-177 (2013).

\section{Acknowledgments}

We thank the members of Britton Chance Center for Biomedical Photonics for advices. This work is supported by the National Nature Science Foundation of China (Nos. 81127002, 30925013, 91232306), and Science Fund for Creative Research Group of China (Grant No. 61121004)

\section{Author contributions}

S.Z. and H.G. conceived the project. S.Z. and T.Q. design the model, and wrote the manuscript. T.Q. and J.L. developed the algorithm, H.Z. wrote software, T.Q., S.L., J.L. and H.Z. performed the image analysis and processing. T.Z., S.Z. and Q.L. design the imaging system, H.G., Z.Y. and T.Z. performed sample preparation.

\section{Additional information}

Competing financial interests: The authors declare no competing financial interests.

How to cite this article: Quan, T.W. et al. Digital reconstruction of the cell body in dense neural circuits using a spherical-coordinated variational model. Sci. Rep. 4, 4970; DOI:10.1038/srep04970 (2014) 
(i) This work is licensed under a Creative Commons Attribution-NonCommercialNoDerivs 3.0 Unported License. The images in this article are included in the article's Creative Commons license, unless indicated otherwise in the image credit; if the image is not included under the Creative Commons license, users will need to obtain permission from the license holder in order to reproduce the image. To view a copy of this license, visit http://creativecommons.org/licenses/by-nc-nd/3.0/ 